\title{
Telmatobius philippii, una nueva especie de rana acuática de Ollagüe, norte de Chile (Leptodactylidae)
}

\author{
Telmatobius philippii, a new species of aquatic frog from Ollagüe, northern Chile \\ (Leptodactylidae)
}

\author{
CÉSAR C. CUEVAS \& J. RAMÓN FORMAS
}

Instituto de Zoología, Universidad Austral de Chile, Casilla 567, Valdivia, Chile; e-mail:ccuevas@uach.cl

\begin{abstract}
RESUMEN
Se describe una nueva especie de rana acuática, Telmatobius philippii, de las proximidades de Ollagüe, II Región, Cordillera de Los Andes, norte de Chile. El nuevo taxón se caracteriza por su tamaño moderado (46,10-53,70 mm), flancos granulares, lengua ovalada sin escotadura; adherida anteriormente al piso de la boca en $75 \%$ de su longitud, dientes maxilares y premaxilares presentes y renacuajos con sistema de la línea lateral conspicua. La descripción de la nueva especie se completa con información osteológica, cromosómica ecológica y larvaria. Telmatobius philippii es comparada con otras especies chilenas del género Telmatobius.
\end{abstract}

Palabras clave: Telmatobius philippii, Ollagüe, norte de Chile.

\begin{abstract}
A new species of aquatic frog, Telmatobius philippii, is described in the proximity of Ollaguie, II Región, Andes range, northern Chile. The new taxon has moderate-sized $(46.10-53.70 \mathrm{~mm})$, granular flanks, tongue oval, unnotched; attached anteriorly about $75 \%$ of its length, maxillary and premaxillary teeth present, tadpole with lateral line system conspicuous. The description of the new species is completed with, osteological, chromosomic, ecological and larval information. Telmatobius philippii is compared with other Chilean species of the genus Telmatobius.
\end{abstract}

Key words: Telmatobius philippii, Ollagüe, northern Chile.

\section{INTRODUCCIÓN}

Los altos Andes al sur de la línea del Ecuador se caracterizan por una extensa radiación de ranas acuáticas del género Telmatobius Wiegmann 1835 (aproximadamente 50 especies; Frost 1985 , Duellman 1993). Altitudinalmente se encuentran sobre los $2.500 \mathrm{~m}$ (excepto T. ceiorum, $1.500 \mathrm{~m}$ ) y longitudinalmente se extienden desde la línea ecuatorial (T. niger) hasta $\operatorname{los} 29^{\circ} \mathrm{S}$, en $\operatorname{los}$ faldeos orientales de los Andes argentinos ( $T$. contrerasi) (Cei 1977).

Vellard (1951) y Cei (1962) citan para Chile a T. halli y T. peruvianus. Veloso \& Navarro (1988) reconocen además a $T$. marmoratus, $T$. pefauri y T. zapahuirensis. Recientemente Formas et al. (1999) describieron a $T$. dankoi en las cercanías de la ciudad de Calama (región de Antofagasta, provincia de El Loa); siendo esta especie el taxón más sureño de Telmatobius descrito para Chile. La escasa exploración batracológica de los altos Andes chilenos, especialmente en las áreas com- prendidas entre las regiones de Tarapacá y Atacama y la presencia de $T$. contrerasi hasta los $29^{\circ} \mathrm{S}$ en la vertiente oriental andina, hacen presumir la existencia de nuevas poblaciones de Telmatobius en esta extensa área geográfica.

En el presente trabajo se describe una nueva especie de Telmatobius proveniente de las cercanías de Ollagüe (provincia de El Loa, región de Antofagasta) y su descripción se acompaña con información osteológica, larvaria, y cromosómica. La nueva especie se compara con otros taxa congenéricos descritos para el territorio chileno.

\section{MATERIALES Y MÉTODOS}

Se examinaron 38 especímenes de Telmatobius, de los cuales 19 pertenecen al nuevo taxón (Anexo 1). Los animales se midieron con un pié de metro de $0,1 \mathrm{~mm}$ de precisión. En los adultos se consideraron nueve variables: (1) distancia hocicocloaca, (2) largo de la cabeza, (3) ancho de la 
cabeza, (4) distancia interorbital, (5) distancia entre las narinas, (6) diámetro del ojo, (7) distancia ojo-narinas, (8) largo de la tibia, y (9) largo del pie. Las medidas se tomaron de acuerdo a Cei (1962, 1980) y Duellman (1970). Para determinar la extensión de la membrana entre los ortejos se usó la fórmula de Savage \& Heyer (1967) modificada por Myers \& Duellman (1982). Los estados larvarios se determinaron según Gosner (1960). Para las larvas se consideraron ocho variables: (1) largo total, (2) largo del cuerpo, (3) alto del cuerpo, (4) alto de la aleta, (5) distancia narina-hocico, (6) distancia narina-ojo, (7) diámetro del ojo y (8) ancho del disco oral. Las medidas larvarias se tomaron de acuerdo a Formas (1992). El estudio osteológico del nuevo taxón está basado en el examen de tres esqueletos [IZUA (Instituto de Zoología de la Universidad Austral de Chile) 3193 , 3194, 3195] teñidos y diafanizados según Dingerkus \& Uhler (1977). Los huesos carpales se identificaron según Fabrezi \& Alberch (1996).

Para el estudio de los cromosomas se utilizaron dos machos y tres hembras (IZUA 3193, 3194, 3195, 3196, 3197); los cariotipos se obtuvieron según la metodología usada por Cuevas y Formas (1996), y los patrones de bandeo C con el método descrito por Sumner (1972). Las posiciones centroméricas fueron determinadas de acuerdo a Levan et al. (1964) y la longitud relativa según Bogart (1970). Todos los especímenes y las preparaciones microscópicas fueron depositados en la colección de anfibios del Instituto de Zoología de la Universidad Austral de Chile.

\section{RESULTADOS}

\section{Sistemática}

Telmatobius philippi: Holotipo IZUA 3093, un macho adulto (Fig. 1A) colectado por César Cuevas, Ramón Formas y Carlos Jara, el 8 de marzo de 1998; Quebrada de Amincha (35 $28^{\prime} \mathrm{S}$, $71^{\circ} 11^{\prime} \mathrm{O}, 3.800 \mathrm{~m}$ de altitud), $7 \mathrm{~km}$ al $\mathrm{NO}$ de Ollagüe, 196 km al NE de Calama (por carretera), provincia El Loa, región de Antofagasta, cordillera de Los Andes, Chile (Fig. 2).

Paratipos. Cinco animales adultos (IZUA 3088, 3089, 3090, 3091, 3092), colectados en la Quebrada del Inca, aproximadamente $1 \mathrm{~km}$ al norte de la localidad típica.

La asignación genérica del nuevo taxón se basa en los criterios de Trueb (1979) y Wiens (1993), quienes indican que Telmatobius se podría definir por la inserción de los dientes maxilares en la mucosa labial, espinulación nupcial sólo en el primer dedo y frontoparietales fusionados.
Diagnosis. Telmatobius philippii puede ser distinguido de sus congéneres por la siguiente combinación de caracteres: (1) En los machos la longitud hocico-cloaca es de 53,70 mm, en las hembras 46,1 mm; (2) flancos con numerosos gránulos pequeños que se extienden ligeramente a las regiones ventrales y superficie dorsal de las extremidades posteriores, (3) lengua oval sin escotadura posterior, adherida al piso de la boca en 3/4 de su longitud, (4) ausencia de pliegues postfemorales, (5) dientes premaxilares y maxilares presentes, dientes vomerianos rudimentarios o ausentes, (6) articulación tibio-tarsal no alcanza el borde posterior del ojo, (7) ausencia de espinulación nupcial en el pecho y brazos, (8) larva con línea lateral conspicua, (9) en vivo el vientre es de color bronce con tonalidades doradas, (10) espinas nupciales en la superficie dorsal y borde posterior del I dedo; no se extienden a la superficie ventral.

Descripción del holotipo. Distancia hocico-cloaca 53,3 mm. Cabeza más ancha $(19,5 \mathrm{~mm})$ que larga $(16,0 \mathrm{~mm})$ (Fig. 1B). El largo de la cabeza corresponde al $29,2 \%$ de la distancia hocicocloaca. Ancho máximo del cuerpo (medido en su parte media) corresponde a 1,2 veces el ancho de la cabeza. Hocico levemente redondeado, tanto en vista dorsal como lateral (Fig. 1A y 1B). Labio superior sin escotadura media. Canthus rostralis indistinto, región loreal plana en vista frontal. Ojos sobresalientes (5,6 $\mathrm{mm}$ de diámetro), de posición anterodorsolateral (Fig. 1A). Distancia interorbitaria $29,7 \%$ el ancho de la cabeza. Narinas redondeadas con reborde definido y más cerca del borde anterior del ojo que del extremo del hocico. Tímpano ausente y anillo timpánico pequeño. Pliegue postocular definido; se extiende desde el borde posterior del ojo hasta el nacimiento del brazo, pero no lo alcanza. Dientes vomerianos ausentes. Dientes maxilares y premaxilares insertos en la mucosa labial. Coanas ovales grandes. Lengua oval, sin escotadura posterior; adherida al piso de la boca en $75 \%$ de su longitud. Dedos delgados, con un pequeño reborde cutáneo y de punta redondeada (Fig. 1C). Fórmula digital III, IV, I, II. Tubérculos subarticulares en todos los dedos con la fórmula I(1), II(1), III(2), IV(2). Tubérculo palmar interno largo y ovalado; palmar externo oval y más corto que el interno. Numerosos tubérculos palmares pequeños. Espinas nupciales pequeñas en la superficie dorsoposterior del primer dedo; no se extienden hacia la superficie ventral (Fig. 1D). Miembros posteriores delgados, 72,7 $\mathrm{mm}$ de longitud. Articulación tibio-metatarsal, extendida anteriormente, no alcanza el borde posterior del ojo. El largo del pie $(29,9 \mathrm{~mm})$ representa el $56,1 \%$ de la longitud 

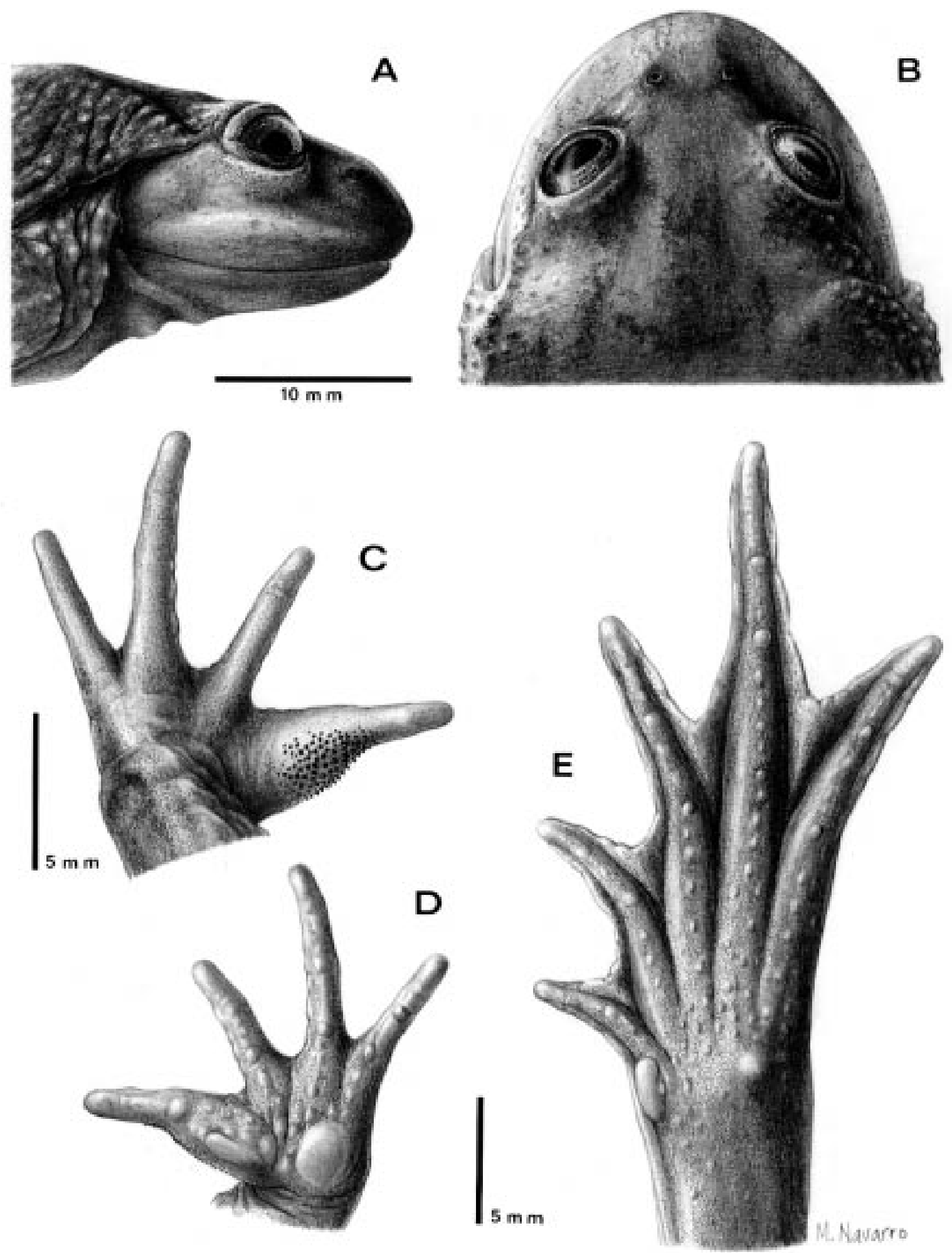

Fig. 1: Holotipo de Telmatobius philippii (IZUA 3093). Vista lateral (A), dorsal (B) de la cabeza, callosidades nupciales (C), superficie palmar (D) y superficie plantar (E).

Holotype of Telmatobius philippii (IZUA 3093). Lateral (A) and dorsal (B) view of head, nuptial asperities (C), palmar surface (D), and plantar surface (E). 
hocico-cloaca, y el largo de la tibia $(21,7 \mathrm{~mm})$ el $40,7 \%$. Ortejos delgados con extremos redondeados, ordenados en longitud decreciente IV, III, V, II, I. Borde cutáneo externo en el I y V ortejo. Tubérculo metatarsal externo pequeño y redondeado; interno alargado y 3,5 veces más grande que el metatarsal externo. La fórmula de los tubérculos subarticulares de los ortejos es la siguiente: I(1), II(1), III(2), IV (3), V(2). Numerosos tubérculos plantares pequeños. Fórmula de las membranas entre los ortejos: I $1 \frac{1}{3}-2 \frac{1}{2}$ II $1 \frac{1}{2}$ 3 III $2 \frac{1}{4}-3 \frac{1}{2}$ IV $3 \frac{1}{2}-1 \frac{1}{4}$ V. Pliegue tarsal evidente, alcanza la articulación tibio-tarsal (Fig. 1 E). Superficie dorsal del miembro posterior con espinulación notoria; superficie ventral sin espinas. Ausencia de pliegues postfemorales. Vientre y garganta sin espinas. Dorso del brazo y antebrazo con espinas pequeñas. Flancos con espinas notorias que se extienden brevemente hacia el dorso y vientre; allí son diminutas. Cabeza carente de espinulación.

Variabilidad. La variabilidad de los caracteres morfométricos de la serie tipo se indican en la Tabla 1. El holotipo (un macho adulto) tiene un grueso pliegue cutáneo detrás de la articulación maxilar (Fig. 1A), además el pliegue postocular y las espinas de los flancos tienen mayor desarrollo que los otros especímenes de la serie tipo. En los especímenes IZUA 3088 y 3089 se presenta un solo diente vomeriano ubicado en el borde posterior de las coanas.

Coloración. El holotipo (IZUA 3093), y los paratipos (IZUA 3088, 3089, 3090, 3092), preservados en alcohol (70 \%) tienen el dorso pardusco con pequeñas manchas negras redondeadas, iguales al diámetro del ojo; el vientre es crema amarillento (excepto el paratipo IZUA
3089, que tiene el vientre con diminutas manchas más obscuras). La garganta posee pequeñas manchas de color crema en todos los especímenes. Las áreas ventrales de la parte anterior de los brazos y piernas, así como la punta de los dedos, ortejos, tubérculos subarticulares, palmares y metatarsales blanco cremoso. En vivo el dorso es verde obscuro con manchas negras; el vientre es de color bronce con tonos dorados.

Etimología. El nuevo taxón es nombrado en homenaje al naturalista Rodulfo Amando Philippi, quien hizo valiosos aportes al estudio de los anfibios chilenos al escribir el "Suplemento a los Batraquios Chilenos, descritos en la Historia Física i Política de don Claudio Gay".

\section{Larva}

Descripción. Basada en una larva (IZUA 3087) colectada en la localidad típica y una serie de ocho renacuajos (IZUA 3094) colectados en la Quebrada del Inca. Cuerpo ovoide en vista lateral y dorsal, 2,3 veces mas largo que alto. Cola robusta con miómeros bien desarrollados. Perfil del hocico redondeado (Fig. 3A). Narinas ovaladas con reborde cutáneo definido; situadas mas cerca del borde anterior del ojo que la punta del hocico y ubicadas anterodorsolaterales. Ojos anterodorsolaterales, y su diámetro anterior es 1,6 veces la distancia internarial. Distancia interocular 1,3 veces la distancia internarial (Fig. 3B). Pupilas circulares con el iris gris dorado. Disco oral translúcido, de posición ventral y transangular; 1,4 veces el diámetro de la distancia interocular. Disco oral con una fila marginal de papilas (excepto en la región rostral) (Fig. 3C).

\section{TABLA 1}

Telmatobius philippii sp. nov., datos morfométricos (mm) de los tipos

Telmatobius philippii sp. nov., morphometric data (mm) of the types

\begin{tabular}{|c|c|c|c|c|c|c|}
\hline \multirow[t]{2}{*}{ Carácter } & \multirow{2}{*}{$\begin{array}{c}\text { Holotipo } \\
\text { IZUA } \\
3093\end{array}$} & \multicolumn{5}{|c|}{$\begin{array}{l}\text { Paratipos } \\
\text { IZUA }\end{array}$} \\
\hline & & 3088 & 3089 & 3090 & 3091 & 3092 \\
\hline Longitud hocico-cloaca & 53,70 & 45,51 & 41,49 & 46,06 & 45,01 & 43,22 \\
\hline Longitud de la cabeza & 17,59 & 17,32 & 17,42 & 18,94 & 18,19 & 16,99 \\
\hline Ancho de la cabeza & 19,93 & 16,85 & 16,42 & 18,07 & 17,70 & 17,05 \\
\hline Longitud de la pierna & 23,16 & 21,76 & 20,32 & 20,38 & 20,85 & 20,41 \\
\hline Longitud de la tibia & 21,86 & 19,59 & 18,54 & 18,73 & 18,81 & 19,07 \\
\hline Longitud del pié & 29,86 & 30,60 & 27,56 & 29,32 & 28,99 & 29,53 \\
\hline Diámetro del ojo & 5,03 & 4,31 & 4,07 & 4,65 & 4,39 & 3,78 \\
\hline Distancia interorbital & 7,29 & 6,14 & 6,15 & 6,18 & 6,58 & 6,47 \\
\hline Distancia hocico-nariz & 4,74 & 3,90 & 3,60 & 4,07 & 3,81 & 3,56 \\
\hline Distancia internarial & 3,64 & 3,06 & 2,80 & 3,11 & 3,25 & 3,16 \\
\hline
\end{tabular}


Papilas presentes en la región supraangular (910) e infraangular (4-6). Rostrodontos más anchos que altos. Suprarostrodontos e infrarostrodontos bien queratinizados con bordes finamente aserrados; pigmentación café oscura. Fórmula de los queratodontos [(1) (1-1)/(1) (1)] (Fig. 3C). Queratodontos curvados y de color café oscuro. Espiráculo ubicado a la izquierda, pegado al cuerpo, y con abertura oblicua ventrodorsal, levemente visible en vista dorsal y con abertura oval (Fig. 3B). Tubo proctodeal traslúcido de abertura ancha y dirigida hacia la derecha (Fig. 3D), su diámetro es casi igual al del ojo. Longitud de la cola 1,5 veces la longitud del cuerpo; eje rectilineo y extremo redondeado. Aleta dorsal sobrepasa levemente el cuerpo y se prolonga levemente por encima de él (Fig. 3A). La aleta ventral nace en el extremo del tubo proctodeal y no sobrepasa la línea ventral del cuerpo. Aletas dorsal y ventral traslúcidas con pequeños y abundantes melanóforos; extremo distal de la cola con abundantes melanóforos (Fig. 3A). Superficie dorsal del cuerpo gris (formalina $10 \%$ ) con pequeños melanóforos. Poros del sistema de la línea lateral notorios y pigmentados de negro. Vientre transparente y asas intestinales visibles. Color en vida similar a los especímenes fijados. La larva descrita se asignó a Telmatobius philippii debido a que esta fue la única especie de anuro colectada en toda el área. Las medidas de las larvas se muestran en la Tabla 2.

Distribución y ecología. Telmatobius philippii se conoce sólo en dos localidades: Quebrada de Amincha (localidad típica, Fig. 2) y Quebrada del Inca; ambas separadas entre sí por aproximadamente $1 \mathrm{~km}$. Los lugares referidos están muy

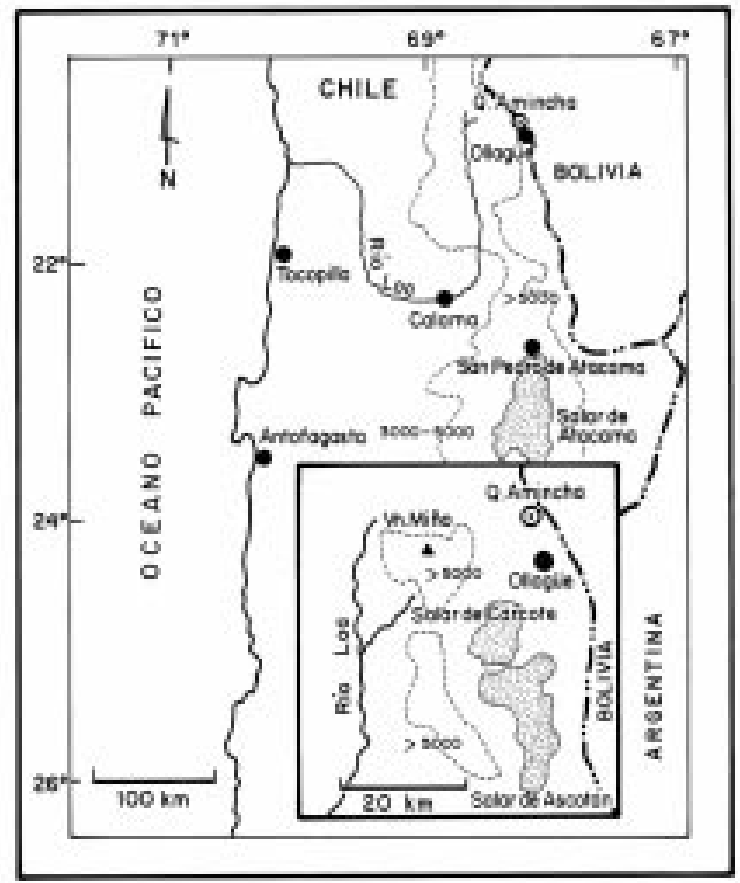

Fig. 2: Localidad típica de Telmatobius philippii. Type locality of Telmatobius philippii.

cerca $(7 \mathrm{~km})$ del pueblo de Ollagüe $(3.700 \mathrm{~m})$ y se sitúan en la región andina descrita por di Castri (1968). En Ollagüe la temperatura promedio anual es de $6,8^{\circ} \mathrm{C}$ [mínima anual $1,9^{\circ} \mathrm{C}$ (julio); máxima anual $10,2^{\circ} \mathrm{C}$ (diciembre y enero)] y el promedio anual de precipitaciones es de 70,6 mm [mínimo anual 0,0 mm (octubre); máximo anual 31,8 $\mathrm{mm}$ (enero)] (Hajek \& di Castri 1975). Los especímenes (adultos y larvas) de T. philippi fue-

TABLA 2

Telmatobius philippii sp. nov., datos morfométricos (media \pm DE y rangos) de las larvas (todos los datos en $\mathrm{mm}$ )

Telmatobius philippii sp. nov., morphometric data (mean \pm SD and ranges) of the tadpoles (all data in $\mathrm{mm}$ )

\begin{tabular}{lccccc}
\hline Carácter & \multicolumn{5}{c}{ Estado } \\
& $\begin{array}{c}25 \\
\mathrm{n}=1\end{array}$ & $\mathrm{n}=2$ & $\begin{array}{c}30 \\
\mathrm{n}=3\end{array}$ & $\begin{array}{c}31 \\
\mathrm{n}=1\end{array}$ & $\begin{array}{c}33 \\
\mathrm{n}=1\end{array}$ \\
\hline Longitud total & 41,22 & $56,24-55,00$ & $63,07 \pm 0,72$ & 72,03 & 85,40 \\
Longitud del cuerpo & 18,00 & $23,14-22,55$ & $26,24 \pm 0,14$ & 29,36 & 35,83 \\
Alto del cuerpo & 7,84 & $10,96-10,74$ & $12,38 \pm 0,65$ & 14,01 & 14,09 \\
Alto de la aleta & 9,41 & $12,17-12,52$ & $13,02 \pm 0,36$ & 14,91 & 14,93 \\
Distancia ojo-hocico & 4,97 & $6,83-6,43$ & $7,65 \pm 0,31$ & 8,68 & 10,34 \\
Distancia nariz-hocico & 3,51 & $4,19-4,14$ & $4,93 \pm 0,15$ & 5,31 & 8,16 \\
Distancia internarial & 2,75 & $2,81-2,92$ & $3,21 \pm 0,32$ & 3,45 & 4,72 \\
Diámetro del ojo & 1,70 & $1,83-1,65$ & $1,68 \pm 0,13$ & 2,01 & 2,79 \\
Distancia interorbital & 3,25 & $3,90-3,92$ & $4,59 \pm 0,25$ & 5,13 & 5,88 \\
Diámetro de la boca & 3,14 & $4,70-4,24$ & $4,76 \pm 0,68$ & 5,52 & 6,71 \\
\hline
\end{tabular}




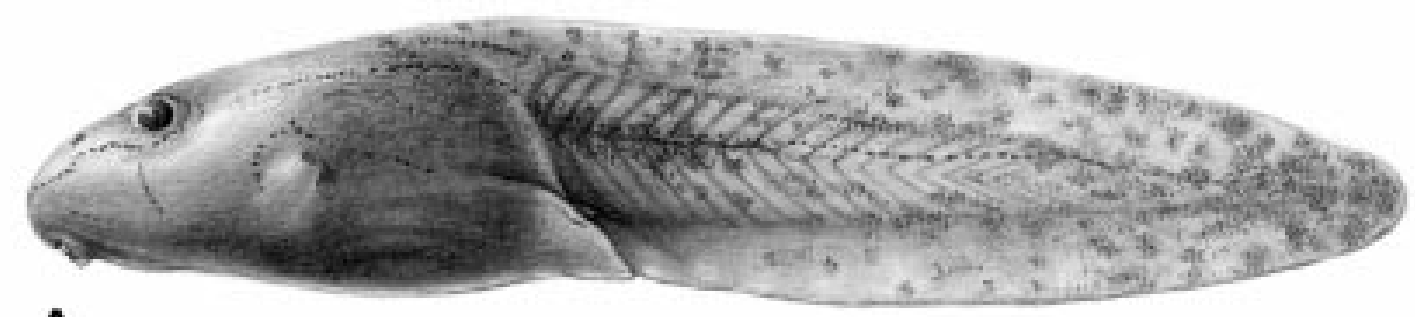

A

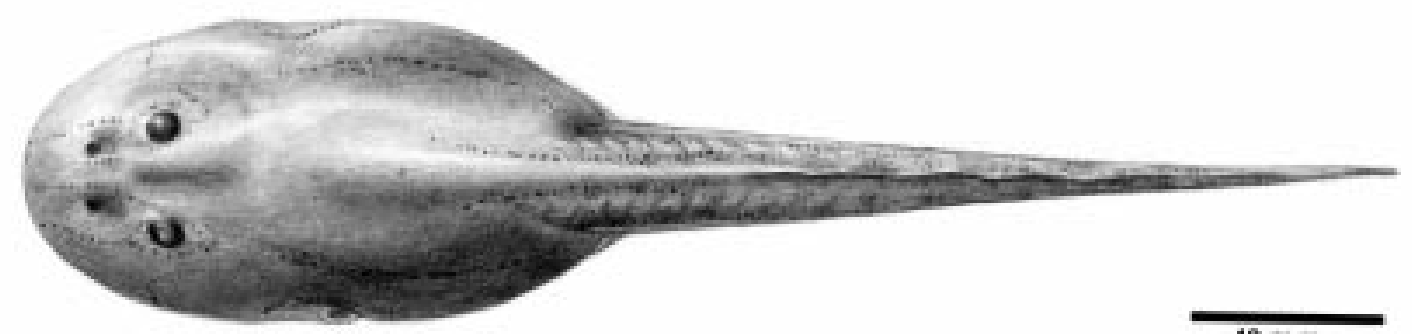

B

$10 \mathrm{~mm}$
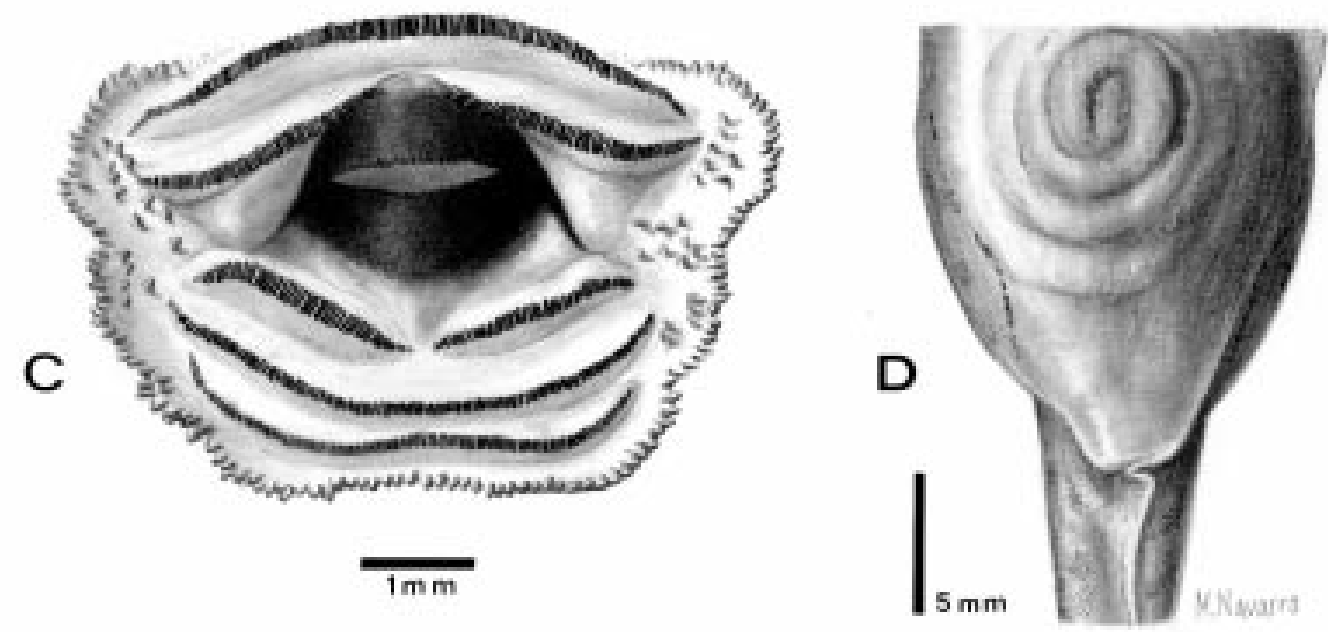

Fig. 3: Vistas lateral (A) y dorsal (B) de la larva (estado 35) de Telmatobius philippii. Disco oral (C) y abertura de la cloaca (D).

Lateral (A) and dorsal (B) views of the tadpole (stage 35) of Telmatobius philippii. Oral disc (C), cloacal aperture (D).

ron colectados en remansos de arroyos $(2-3 \mathrm{~m}$ de ancho, 30 a $40 \mathrm{~cm}$ de profundidad, y 8 a $10{ }^{\circ} \mathrm{C} \mathrm{de}$ temperatura) con abundante vegetación (Ciperaceae). En el agua se encontraron algas de los géneros Spirogyra (Clorophyta) y Chara (Charophyta). Entre la fauna acuática se observaron algunos coleópteros de la familia Dytiscidae. En el contenido estomacal de una hembra adulta (IZUA 3194) se identificaron sólo dos ítems: una larva de mariposa (Noctuidae) y una larva de libélula (Gomphidae). En la fecha de recolecta ( 8 marzo 1998) se encontró un macho con espinu- lación sexual bien desarrollada y hembras con oocitos (257) de 1,6-1,7 mm de diámetro; los que tenían abundante pigmentación melánica en el polo animal.

\section{Cromosomas}

Basados en el análisis de 10 placas metafásicas se determinó que Telmatobius philippii posee un número diploide de $2 n=26$. Todos los cromosomas tienen dos brazos y el número fundamental (NF) 
es 52. Cuando los cromosomas son ordenados en pares de longitud decreciente, los pares 1-4 son grandes (> 100 unidades), el par 5 es intermedio (entre 80 y 100 unidades), y los pares $6-13$ pequeños (< 80 unidades) (Fig. 4A). De acuerdo a la razón (r) de los brazos cromosómicos ( $\mathrm{r}=$ longitud brazo largo/longitud brazo corto), los pares 1 , $3,7,8,9,10,11,12$ y 13 son metacéntricos $(\mathrm{m})$. Pares 4 y 6 son submetacéntricos (sm), y los pares 2 y 5 son subtelocéntricos (st). En el brazo corto de ambos homólogos del par 6 existe una constricción secundaria en posición submetacéntrica. Las medidas de los cromosomas se muestran en la Tabla 3. El cariotipo bandeado (bandas
C) (Fig. 4B) muestra heterocromatina centromérica en todos los cromosomas, excepto en el par 1. Bandas teloméricas C están presentes en los brazos largos de los pares cromosómicos 1, 4, 7 y 8 . Una suave banda telomérica está presente en el brazo corto de ambos homólogos del par 1, y una conspicua banda en esta misma posición del par 7. Una banda $C$, intersticial, se encuentra ubicada en los brazos cortos del par 6 asociada a la región NOR (Fig. 4C). En la Fig. 5 se muestra un idiograma con el patrón de bandeo C. En los cariotipos de $T$. philippii no se detectaron cromosomas sexuales.

\section{A}
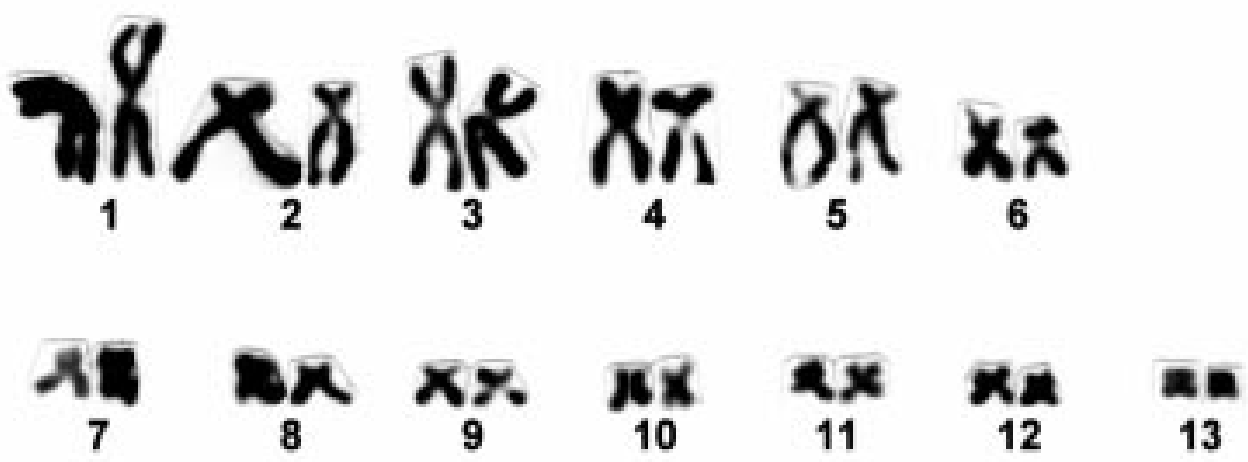

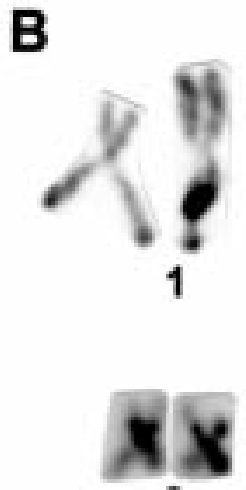

6

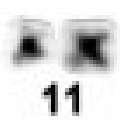

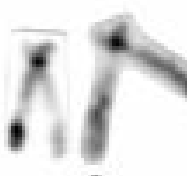

2

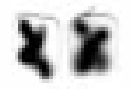

7

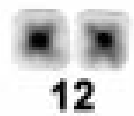

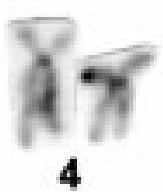

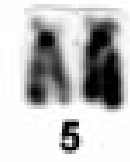

C
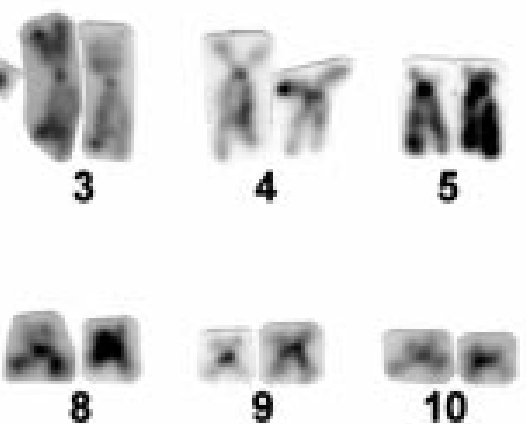

Fig. 4: Cromosomas de Telmatobius philippii. Cariotipo (A), patrón de bandeo C (B) y organizadores nucleolares (NOR) (C).

Chromosomes of Telmatobius philippii. Karyotype (A), C-banded pattern (B) and nucleolar organizer region (NOR) (C). 
TABLA 3

Telmatobius philippii sp. nov., datos cariológicos (media \pm DE)

Telmatobius philippii sp. nov., karyotype data (mean $\pm \mathrm{SD})$

\begin{tabular}{lccc}
\hline Par & Longitud relativa $^{\mathrm{a}}$ & $\mathrm{r}^{\mathrm{b}}$ & Tipo $^{\mathrm{c}}$ \\
\hline 1 & $163,75 \pm 2,08$ & $1,09 \pm 0,02$ & $\mathrm{~m}$ \\
2 & $133,58 \pm 1,50$ & $1,77 \pm 0,31$ & $\mathrm{sm}$ \\
3 & $116,29 \pm 1,29$ & $1,40 \pm 0,18$ & $\mathrm{~m}$ \\
4 & $104,55 \pm 1,21$ & $1,83 \pm 0,68$ & $\mathrm{sm}$ \\
5 & $94,11 \pm 0,61$ & $3,13 \pm 0,37$ & $\mathrm{st}$ \\
$6^{*}$ & $77,14 \pm 1,11$ & $2,66 \pm 0,60$ & $\mathrm{sm}$ \\
7 & $59,37 \pm 0,71$ & $1,52 \pm 0,31$ & $\mathrm{~m}$ \\
9 & $50,31 \pm 0,40$ & $1,38 \pm 0,14$ & $\mathrm{~m}$ \\
10 & $47,87 \pm 0,31$ & $1,32 \pm 0,16$ & $\mathrm{~m}$ \\
11 & $43,30 \pm 0,51$ & $1,31 \pm 0,13$ & $\mathrm{~m}$ \\
13 & $41,26 \pm 0,47$ & $1,30 \pm 0,17$ & $\mathrm{~m}$ \\
\hline
\end{tabular}

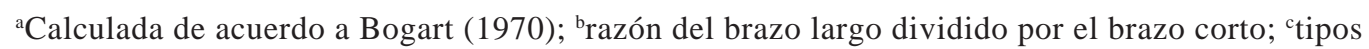
cromosómicos $(\mathrm{m}=$ metacéntrico; $\mathrm{r}=1.0-1.7, \mathrm{sm}=$ submetacéntrico; $\mathrm{r}=1.71-3.0, \mathrm{st}=$ subtelocéntrico; $r=3.01-7.0)$ según Levan et al. (1964); *par con la constricción secundaria

${ }^{\mathrm{a} C}$ Calculated according to Bogart (1970); ${ }^{\mathrm{b}}$ ratio of short arm divided into long arm; ${ }^{\mathrm{c}}$ chromosomic types $(\mathrm{m}=$ metacentric; $\mathrm{r}$ $=1.0-1.7, \mathrm{sm}=$ submetacentric $; \mathrm{r}=1.71-3.0, \mathrm{st}=$ subtelocentric $; \mathrm{r}=3.01-4.5)$ according to Levan et al. (1964); *pair with secondary constriction

\section{Osteología}

Cráneo. Cráneo normalmente osificado, 1,1 veces más ancho que largo.

Frontoparietales fusionados posteriormente y en contacto con el proótico y el occipital (Fig.6A). Fontanela frontoparietal abierta anteriormente y tan larga como la longitud anterior de la órbita. Esfenoetmoides mediano, extremo anterior romo; posteriormente (superficie dorsal) en contacto con los frontoparietales y ventralmente cubierto por el paraesfenoides. Nasales bien desarrolla- dos, separados medialmente, arqueados lateralmente y con el borde posterior en contacto con el borde anterior del esfenoetmoides. Arco maxilar completo. Procesos alares de los premaxilares de altura moderada y orientados posteriormente. Premaxilares y maxilares con dientes finos y curvados. Región facial del maxilar inclinada medialmente; proceso preorbital bajo. Proóticos y exooccipitales fusionados, pero medianamente osificados. Cóndilos occipitales sin pedúnculos, medialmente separados. Columela ausente. Procesos óticos y zigomáticos del pterigoides de

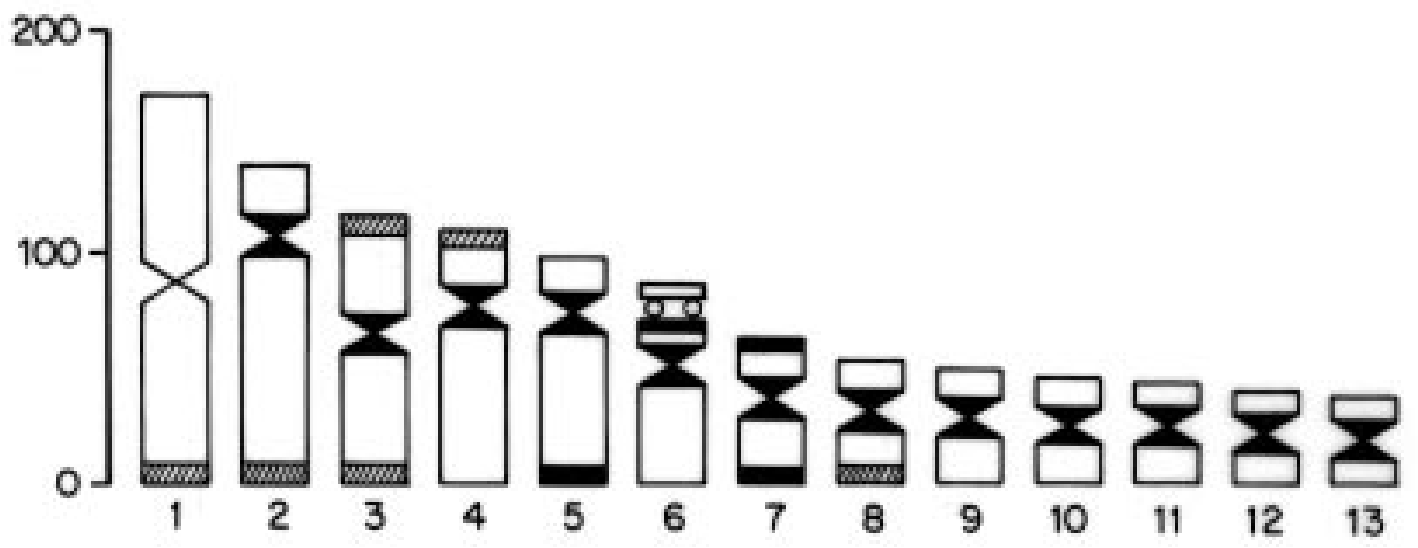

Fig. 5: Idiograma del patrón de bandeo $\mathrm{C}$ de Telmatobius philippii.

C-banded pattern idiogram of Telmatobius philippii. 
desigual longitud; el primero una vez y media más largo que el segundo, y éste último con su extremo posterior ensanchado. El proceso zigomático es puntiagudo e inclinado levemente hacia abajo (Fig. 6C). Angulo máxilo-escamosal de aproximadamente $60^{\circ}$. Paraesfenoides cruciforme (Fig. 6B), su proceso cultriforme alcanza la línea palatina. Posteriormente se sobrepone al exoccipital. Rama anterior del pterigoides en contacto con el zócalo palatino de la maxila. Palatinos delgados y casi rectos, medialmente
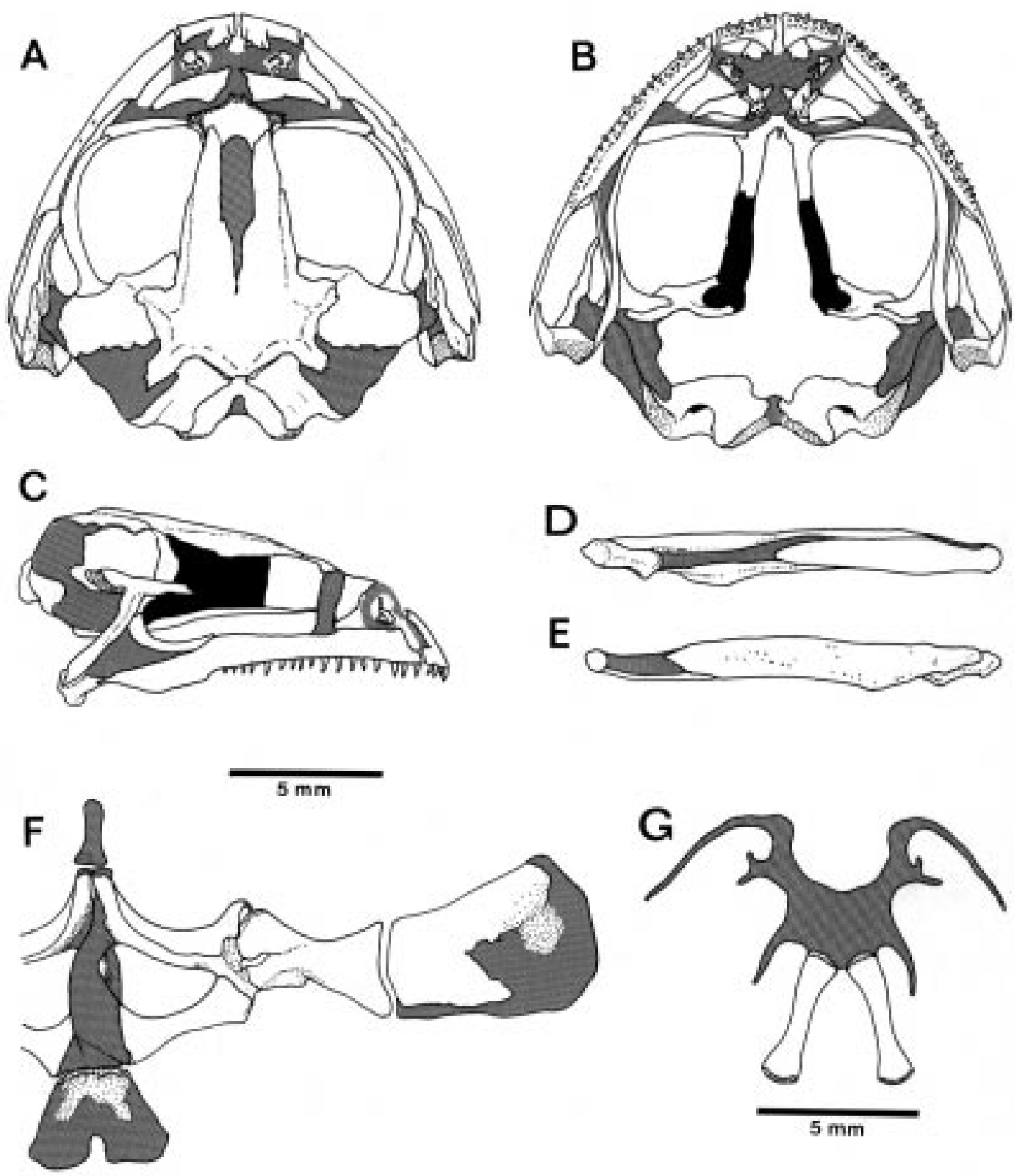

tocan al esfenoetmoides. Vómeres presentes y bien desarrollados; existen rudimentos (4-5 por vomer) de inserciones de odontóforos, pero no se han evidenciado dientes rudimentarios. Mandíbula inferior con cartílago mentomeckeliano osificado; dentario grueso, ángulo espleinal largo (Fig. 6D y 6E). Mandíbulas unidas por un cartílago.

Hyoides. Placa hyoídea cartilaginosa. Seno hipoglosal en forma de U, aproximadamente 1,3 veces más ancho que alto. Procesos alares delga-

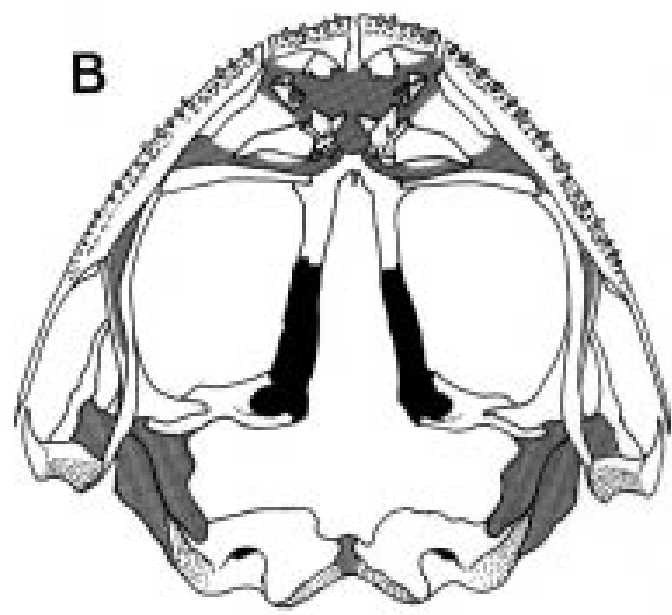

D

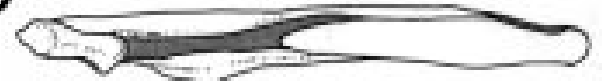

E

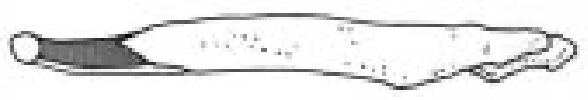

Fig. 6: Vistas dorsal (A), ventral (B) y lateral (C) del cráneo de Telmatobius philippii (IZUA 3193). Vista interna (D) y externa de la mandíbula inferior (E), cintura pectoral (F), hyoides (G).

Dorsal (A), ventral (B) and lateral (C) views of the cranium of Telmatobius philippii (IZUA 3193). Internal (D), and external (E) views of the inferior mandible, pectoral girdle $(\mathrm{F})$, hyoid $(\mathrm{G})$. 
dos, sin expansión distal; posterolaterales delgados. Procesos anteriores del hyal delgados y fuertemente curvados; posteromediales mineralizados, con extremos distales cartilaginosos (Fig. 6G).

Cintura pectoral. Arcífera y poco mineralizada (Fig. 6F). Clavículas arqueadas, no se tocan en el plano mediano; extremos extendidos anteriormen- te hasta la línea que pasa por el borde anterior de las escápulas. Clavículas en contacto con la par acromalis, pero no soldadas a ella. Escápula recta, extremo proximal bicefálico. Supraescápula en forma de paleta, levemente mineralizada en el borde anterior y en el extremo proximal. Coracoides rectos, expandidos en sus extremos.
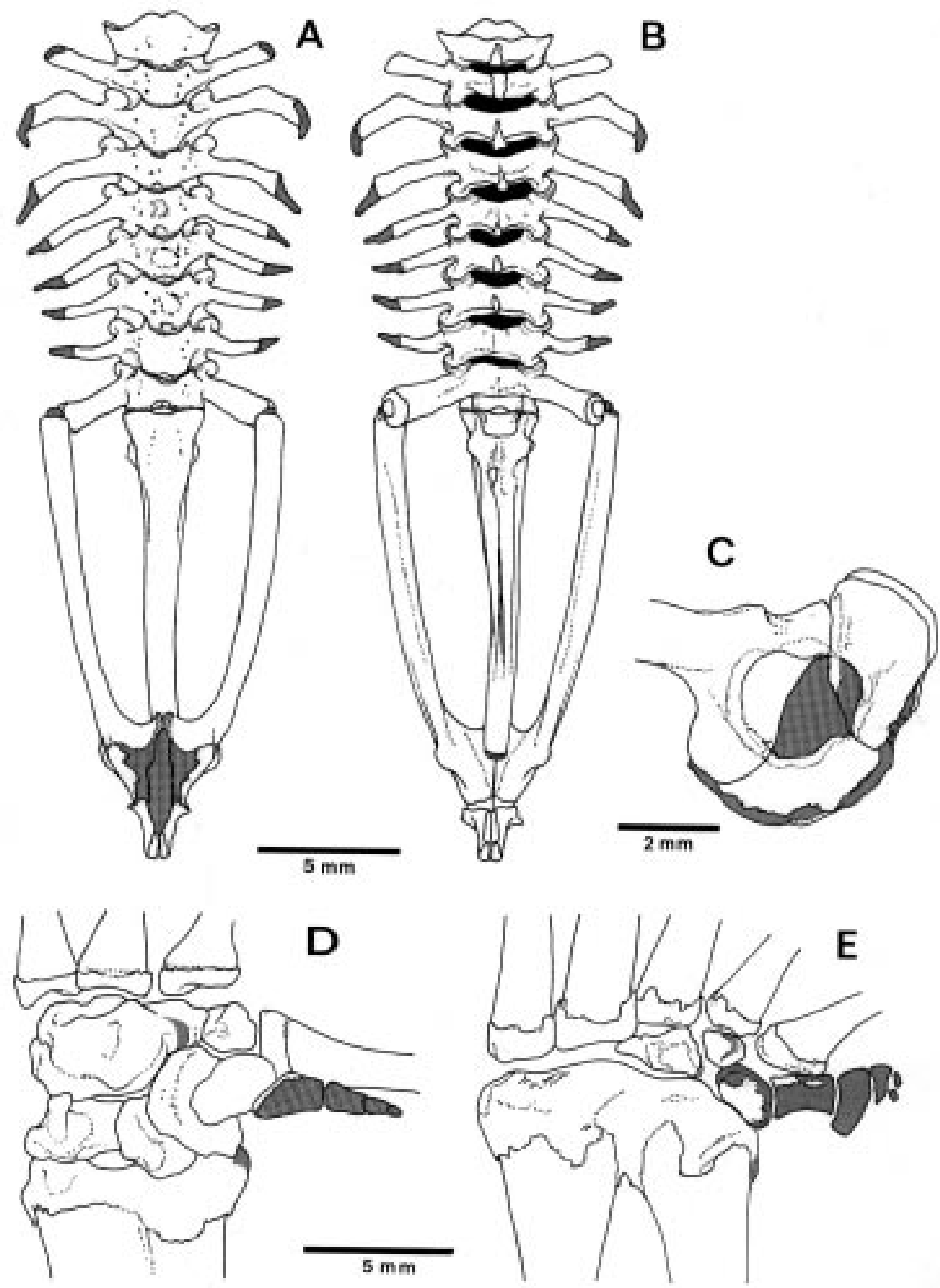

Fig. 7: Vistas dorsal (A) y ventral (B) de la columna vertebral de Telmatobius philippii (IZUA 3193 ). Vista lateral del acetábulo (C), carpo (D), tarso (E).

Dorsal (A), and ventral (B) views of the vertebral column of Telmatobius philippii (IZUA 3193). Lateral view of the acetabulum (C), carpus (D), and tarsus (E). 
Sternum mineralizado en el centro, aproximadamente 1,5 veces más ancho que alto, borde distal hendido en el plano mediano. Xiphisternum corto, cartilaginoso, sin expansión distal.

Columna vertebral. Con ocho vértebras procélicas independientes (Fig. 7A y 7B). Cótilos cervicales moderadamente separados. Arcos neurales no imbricados, con espinas neurales desarrolladas. Vértebras II-IX con procesos transversos y costillas ausentes. Procesos transversos de las vértebras III y IV más grandes que los de las otras vértebras. Extremos distales de los procesos transversos de la vértebra III levemente ensanchados. Procesos transversos II y VIII flectados anteriormente; III -VII de orientación posterior. Porción proximal de la vértebra III flectada anteriormente, pero su extremo distal está dirigida hacia atrás. Articulación sacrococcigea bicondilar; diapófisis sacrales levemente expandidas y de orientación posterior. Coxis con prominencia anterodorsal gruesa en su extremo proximal, luego se transforma en una baja y delgada lámina que desaparece en el tercio posterior.

Cintura pélvica. Cresta ilíaca baja y delgada, desaparece en el tercio anterior. Protuberancia dorsal corta, orientada lateralmente. Expansión acetabular ventral de escaso desarrollo (Fig. 7C); ángulo preacetabular de aproximadamente $90^{\circ}$. Acetábulo redondeado, de bordes delgados y leve escotadura dorsal. Pubis con poca mineralización. Isquio de desarrollo mediano.

Apéndices. Húmero delgado, aproximadamente 1,3 veces mas largo que la radioulna. Cresta anterior delgada, alcanza sólo a la mitad de la cara anterior del húmero. Fórmula de las falanges de la mano: 2-2-3-3; falanges terminales aguzadas. Se identificaron los siguientes elementos carpales: prepollex con cuatro piezas cartilaginosas, distal carpal 2 independiente, Distal Carpal 3-5 fusionado, Elemento Y, radial y ulnar independientes (Fig. 7D). Fémur y tibia iguales en longitud, tibial y fibular fusionados proximal y distalmente. Fórmula de falanges en el pie: 2-2-3-4-3; extremo distal de las falanges puntiagudas. Prehallux con dos elementos grandes bien definidos y tres rudimentos distales, tres huesos metatarsales de homología indefinida (Fig. 7E).

\section{DISCUSIÓN}

Los caracteres morfológicos externos de adultos y larvas, la osteología y las características del cariotipo de $T$. philippii, proporcionan información relevante que justifican la erección del nuevo taxón y su adscripción al género Telmatobius. Telmatobius philippii es la séptima especie del género descrita para Chile y la tercera para la región de Antofagasta ( $T$. halli, $T$. dankoi y $T$. philippii). La presencia de otras dos nuevas especies en los altos Andes de Antofagasta, las que en éste momento están en proceso de descripción por uno de nosotros (J.R. Formas), indica que en esta área habría al menos cinco especies de Telmatobius. Este antecedente adicional sugiere que las áreas altoandinas de la Región de Antofagasta fueron un probable centro de diversificación austral para Telmatobius. Esta proposición debería ser corroborada por nuevos hallazgos de Telmatobius en las Regiones de Antofagasta y Atacama. La presencia de Telmatobius en esta última Región (Atacama) no parece improbable pues en la Argentina se ha reportado la existencia de Telmatobius (T. contrerasi) hasta $\operatorname{los} 29^{\circ} \mathrm{de}$ latitud S (Cei 1977).

Telmatobius philippii se puede diferenciar claramente de las otras especies congenéricas presentes en Chile (T. dankoi, T. halli, T. marmoratus, T. pefauri, T. peruvianus y T. zapahuirensis) por características externas bien definidas. Telmatobius halli se diferencia de T. philippii porque en la primera especie la articulación tibiotarsal toca el borde posterior del ojo, en la segunda nunca lo alcanza; en $T$. halli el hocico es truncado en vista dorsal mientras que en $T$. philippii es redondeado. Telmatobius halli presenta una gran reducción en tamaño y número de los dientes maxilares (Noble 1938), en cambio $T$. philippii tiene dientes maxilares y premaxilares desarrollados. Esta última característica también diferencia a $T$. philippii de $T$. dankoi la que carece de dientes maxilares y vomerianos (Formas et al. 1999). Telmatobius peruvianus posee espinulación nupcial en el pecho, en $T$. philippii las espinas se presentan sólo en la superficie dorsal del primer dedo. En T. marmoratus las membranas entre los ortejos son mas gruesas y tienen buen desarrollo (I $1-1 \frac{3}{4}$ II $1 \frac{1}{2}-2^{3} /{ }_{4}$ III $2 \frac{1}{3}-3 \frac{1}{3}$ IV $3 \frac{1}{2}-$ V), en $T$. philippii son más delgadas y menos extensas (I $1^{1} /$ $3^{-2} \frac{1}{2}$ II $1 \frac{1}{2}-3$ III $2 \frac{1}{4}-3 \frac{1}{2}$ IV $3 \frac{1}{2}-1 \frac{3}{4}$ V). En $T$. philippii el pliegue tarsal alcanza la articulación tibiometatarsiana, mientras que en T. marmoratus sólo llega hasta la mitad del tarso. Telmatobius pefauri es una especie de tamaño mediano (75 $\mathrm{mm}$; distancia hocico cloaca) (Veloso \& Trueb 1976) y su articulación tibio-tarsal no alcanza el borde posterior del ojo (en T. philippii sobrepasa el borde anterior del ojo). Telmatobius zapahuirensis se diferencia de $T$. philippii por tener escasas espinas córneas pequeñas, irregularmente repartidas en el pecho; en $T$. philippii estas espinas están ausentes.

Si bien $T$. philippii y $T$. halli han sido descritas en las cercanías de Ollagüe sus distribuciones 
TABLA 4

Características cromosómicas de algunas especies del género Telmatobius

Chromosomes characteristics of some species of the genus Telmatobius

\begin{tabular}{|c|c|c|c|c|c|}
\hline Especie & $2 n^{a}$ & $\mathrm{NF}^{\mathrm{b}}$ & $\mathrm{CS}^{\mathrm{c}}$ & Tipos & Autores \\
\hline T. arequipensis & 26 & 52 & 6 & m. sm. st. & Córdoba et al. (1987) \\
\hline T. carrillae & 26 & 52 & -- & -- & Morales (1988) \\
\hline T. ceiorum & 26 & 52 & -- & -- & Morescalchi (1973) \\
\hline T. dankoi & 26 & 52 & 6 & m. sm. st. & Formas et al. (1999) \\
\hline T. marmoratus & 26 & 52 & 6 & m. st. & Veloso et al. (1982) \\
\hline T. sp. grupo marmoratus & 22 & 44 & -- & m. sm. & Brum-Zorrilla \& Sáez (1968) \\
\hline T. schreiteri & 26 & 52 & -- & -- & Barbieri (1954) \\
\hline T. stephani & 26 & 52 & -- & -- & Morescalchi (1973) \\
\hline T. pefauri & 26 & 52 & 6 & m.sm.st. & Veloso et al. (1982) \\
\hline T. peruvianus & 26 & 52 & 6 & m.sm.st. & Veloso et al. (1982) \\
\hline T. philippii & 26 & 52 & 6 & m.sm.st. & Este trabajo \\
\hline T. zapahuirensis & 26 & 52 & 6 & m.sm.st. & Veloso et al. (1982) \\
\hline
\end{tabular}

${ }^{a}$ Número diploide; ${ }^{b}$ número fundamental de brazos; ' posición de la constricción secundaria

altitudinales son diferentes. Telmatobius philippii ha sido colectada en un arroyo $\left(8-10^{\circ} \mathrm{C}\right)$ a $3.800 \mathrm{~m}$, mientras que $T$. halli fue capturada en un pozo de aguas termales a $3.048 \mathrm{~m}$ (10.000 pies, Noble 1938).

La larva de T. philippii pertenece al tipo generalizado de Orton (1953), y su descripción concuerda con las características dadas por Lavilla (1988) para las larvas del género Telmatobius. La ausencia de papilas en la región intramarginal mental de la larva, sustenta la ubicación de $T$. philippii en el grupo septentrional (Lavilla 1988), compuesto por especies bolivianas (T. culeus), chilenas (T. halli, T. pefauri), ecuatorianas (T. niger, T. vellardi) y peruanas (T. albiventris, T. arequipensis, $T$. crawfordi, T. jelskii, T. marmoratus T. peruvianus, T. rimac). Otras especies argentinas ( $T$. atacamensis, T. ceiorum, T. hauthali, T. laticeps, T. oxicephalus, T. schreiteri, T. stephani) tienen larvas con papilas en la región intramarginal mental (Lavilla 1988). La presencia en la larva de una conspicua línea lateral ha sido solamente reportada en $T$. philippii.

Telmatobius philippii posee 26 cromosomas (NF =52), como en la mayoría de las especies congenéricas. (Tabla 4). Brum-Zorrilla \& Sáez (1968) indican que una especie indeterminada de Telmatobius del grupo marmoratus (La Paz, Bolivia) posee 22 cromosomas. Veloso et al. (1982) señalan que Telmatobius marmoratus procedente del norte de Chile (Parinacota) tiene un cariotipo con 26 cromosomas. La presencia inusual de la fórmula $2 \mathrm{n}=22$ en un Telmatobius boliviano podría indicar la presencia de al menos dos líneas evolutivas dentro del género; sin embargo, para fundamentar apropiadamente esta suposición es necesario disponer de información cariológica adicional.

El patrón de distribución de la heterocromatina constitutiva (bandas $\mathrm{C}$ ) ha sido reportado sólo en $T$. arequipensis, $T$. dankoi y ahora en $T$. philippii. El cariotipo bandeado de esta última especie difiere de las otras en la presencia de pocas bandas $\mathrm{C}$ teloméricas, y por la presencia de una banda intersticial asociada a la constricción secundaria en el brazo corto del par 6 (sm). Esta comparación sugiere que $T$. philippii posee una menor cantidad de heterocromatina que $T$. arequipensis y $T$. dankoi.

\section{AGRADECIMIENTOS}

Este trabajo fue financiado por el proyecto sectorial FONDECYT S960021. Juan Carlos Ortiz, Alberto Veloso, Patricia Iturra y José Navarro proporcionaron ejemplares de Telmatobius depositados tanto en el Museo de Zoología de la Universidad de Concepción, como en el Departamento de Biología Celular y Genética de la Universidad de Chile. Nuestro especial reconocimiento para el Dr. Carlos Jara S. por su ayuda entusiasta y eficaz en el trabajo de campo. Marcos Navarro elaboró los dibujos. Edgar Benavides hizo los dibujos del holotipo de T. halli los que se compararon con los especímenes de $T$. philippii.

\section{LITERATURA CITADA}

BARBIERI FD (1954) Observaciones preliminares sobre los cromosomas de Telmatobius schreiteri. Scientia Genetica 4: 223-226. 
BOGART JP (1970) Systematic problems in the amphibian family Leptodactylidae (Anura) as indicated by karyotypic analysis. Cytogenetics 9: 369-383.

BRUM-ZORRILLA N \& FA SÁEZ (1968) Chromosomes of Leptodactylidae (Amphibia: Anura). Experientia 24: 969.

CEI JM (1962) Batracios de Chile. Ediciones de la Universidad de Chile, Santiago, Chile. cviii +128 pp.

CEI JM (1977) A new species of Telmatobius from the northern mountains of San Juan, Argentina (Anura: Leptodactylidae). Journal of Herpetology 11: 359361.

CEI JM (1980) Amphibians of Argentina. Monitore Zoologico Italiano N S. Monografía 2. xii + 609 pp.

CÓRDOVA JH, J DESCAILLEAUX \& W MANYA (1987) Descripción del cariotipo de Telmatobius arequipensis (Anura: Leptodactylidae) y relaciones citogenéticas con otras especies del género. Revista Latinoamericana de Genética 1: 44 - 53.

CUEVAS CC \& JR FORMAS (1996) Heteromorphic sex chromosomes in Eupsophus insularis (Amphibia: Anura: Leptodactylidae). Chromosome Research 4: 467-470.

DI CASTRI F (1968) Esquisse écologique du Chili: En: Delamare-Deboutteville CL \& E Rapoport (eds) Biologie de l'Amerique australe: 7- 52. Editions du Centre National de la Recherche Scientifique, Paris, Francia.

DINGERKUS G \& LD UHLER (1977) Enzyme clearing of alcian blue stained whole small vertebrates for demonstration of cartilage. Stain Technology 52: 229232.

DUELLMAN WE (1970) The hylid frogs of the Middle America. Monograph of Museum of Natural History, University of Kansas 1: 1-753.

DUELLMAN WE (1993) Amphibian species of the world: additions and corrections. The University of Kansas, Museum of Natural History, Special Publications, Lawrence, Kansas. iii +372 pp.

FABREZI M \& P ALBERCH (1996) The carpal elements of anurans. Herpetologica 52: 158- 204.

FORMAS JR (1992) The tadpole of Eupsophus vertebralis (Anura: Leptodactylidae). Herpetologica 48: 115-119.

FORMAS JR, I NORTHLAND, J CAPETILLO, JJ NÚÑEZ, CC CUEVAS \& L BRIEVA (1999) Telmatobius dankoi, una nueva especie de rana acuática del norte de Chile (Leptodactylidae). Revista Chilena de Historia Natural 72: 427-445.

FROST DR (1985) Amphibians species of the world: a taxonomic and geographic reference. Allen Press, Inc. and The Associations of Systematics Collections, Lawrence, Kansas. v + 732 pp.

GOSNER KL (1960) A simplified, table for staging anuran embryos and larvae with notes on identification. Herpetologica 16: 183-190.

HAJEK ER \& F DI CASTRI (1975) Bioclimatología de Chile. Dirección de Investigación, Vice-Rectoría Académica Universidad Católica de Chile, Santiago, Chile. 107 pp.
LAVILLA EO (1988) Lower Telmatobiinae (Anura: Leptodactylidae): generic diagnoses based on larval characters. Occasional Papers, Museum of Natural History, University of Kansas 124: 1-19.

LEVAN A, A FREDGA \& A SANDBERG (1964) Nomenclature for centromeric positions on chromosomes. Hereditas 52: 201-220.

MORALES VR (1988) Una nueva especie de Telmatobius (Anura, Leptodactylidae) de Ancash, Perú. Revista Brasileira de Zoología 5: 603-608.

MORESCALCHI A (1973) Amphibia. En: Chiarelli AB \& E Capanna (eds) Cytotaxonomy and vertebrate evolution: 233-348. Academic Press, London, United Kingdom.

MYERS CH \& WE DUELLMAN (1982) A new species of Hyla from Cerro Colorado, and other tree frog records and geographical notes from western Panamá. American Museum of Natural History 2752: 1-32.

NOBLE GK (1938) A new species of frog of the genus Telmatobius from Chile. American Museum Novitates 973: 1-3.

ORTON GL (1953) The systematics of vertebrate larvae. Systematic Zoology 2: 63-75.

SAVAGE JM \& R HEYER (1967) Variation and distribution in the tree frog genus Phyllomedusa in Costa Rica, Central America. Beiträge für Neotropischen Fauna 2: 111-131

SUMNER AT (1972) A simple technique for demonstrating centromeric heterochromatin. Experimental Cell Research 75: 304-306.

TRUEB L (1979) Leptodactylid frogs of the genus Telmatobius in Ecuador, with the description of a new species. Copeia 1979: 714- 733.

VELLARD J (1951) Estudio sobre batracios andinos. I. El grupo Telmatobius y formas afines. Memorias Museo Historia Natural Javier Prado 1: 1-89 + viii láminas.

VELOSO A \& J NAVARRO (1988) Lista sistemática y distribución geográfica de anfibios y reptiles de Chi1e. Bolletino del Museo Regionale di Scienze Naturali, Torino 6: 481-539.

VELOSO A \& L TRUEB (1976) Description of a new species of telmatobiine frog, Telmatobius (Amphibia: Leptodactylidae), from the Andes of northern Chile. Occasional Papers of the Museum of Natural History, The University of Kansas 62: 1-10.

VELOSO A, M SALABERRY, J NAVARRO, P ITURRA, J VALENCIA, M PENNA \& N DÍAZ (1982) Contribución sistemática al conocimiento de la herpetofauna del extremo norte de Chile. En: Oficina Regional de Ciencia y Tecnología de la Unesco para América Latina y el Caribe, ROSTLAC (eds) El Hombre y los ecosistemas de montaña. Volumen I. La vegetación y los vertebrados inferiores de los pisos altitudinales entre Arica y Lago Chungará: 135-268. Montevideo, Uruguay.

WIEGMANN AF (1835) Beiträge zur Zoologie, gesammelt auf einer Reise um die Erde von Dr. F.J.F. Meyen. Siebente abhandlung. Amphibien. Nova Acta Academische Leopoldina Carolinae 17: 183-262.

WIENS JJ (1993) Systematics of the leptodactylid frog genus Telmatobius in the Andes of northern Perú. Occasional Papers of the Museum of Natural History, University of Kansas 162: 1-76.

Editor Asociado: J.C. Torres-Mura

Recibido el 13 de diciembre de 2000; aceptado el 22 de noviembre de 2001 
ANEXO 1

Abreviaturas: American Museum Natural History, New York (AMNH), Departamento de Biología Celular y Genética, Universidad de Chile (DBCG), Instituto de Zoología, Universidad Austral de Chile (IZUA), Museo de Zoología, Universidad de Concepción (MZUC)

Especímenes examinados:

Telmatobius halli: AMNH 44753 (Holotipo), "Warm spring near" Ollagüe, provincia El Loa, 3.048 m, Chile. Telmatobius dankoi: IZUA 2108-2109-2110 (serie tipo, tres especímenes adultos); IZUA 2107 (5 larvas); Las Cascadas, Calama, provincia El Loa, $2.260 \mathrm{~m}$, Chile.

Telmatobius marmoratus: MZUC 24523-24525, alrededores del lago Chungará, provincia de Parinacota, $4.270 \mathrm{~m}$, Chile.

Telmatobius philippii IZUA 3093 (holotipo), IZUA 3087 (una larva); Quebrada de Amincha, 7 km NO de Ollagüe, provincia El Loa, $3.800 \mathrm{~m}$, Chile.

Telmatobius philippii IZUA 3088-3092 (paratipos, cinco adultos), IZUA 3193-3195 (tres esqueletos, cromosomas), IZUA 3196-3197 (dos adultos, cromosomas), IZUA 3094 (siete larvas); 8 km NO de Ollagüe, Quebrada del Inca, provincia El Loa, $3.800 \mathrm{~m}$, Chile.

Telmatobius pefauri: DBCG 633, 634, 652, Zapahuira, provincia de Parinacota, 3.270 m, Chile.

Telmatobius peruvianus: MZUC 24642-24643, Putre, provincia de Parinacota, $3.200 \mathrm{~m}$, Chile.

Telmatobius zapahuirensis: DBCG 629, 630, 639, Zapahuira, provincia de Parinacota, 3.270 m, Chile. 